ARTICLE

\title{
The evolution of cichlid fish egg-spots is linked with a cis-regulatory change
}

M. Emília Santos ${ }^{1, \dagger}$, Ingo Braasch ${ }^{2}$, Nicolas Boileau ${ }^{1}$, Britta S. Meyer ${ }^{1, \dagger}$, Loïc Sauteur ${ }^{3}$, Astrid Böhne ${ }^{1}$, Heinz-Georg Belting ${ }^{3}$, Markus Affolter ${ }^{3} \&$ Walter Salzburger ${ }^{1}$

The origin of novel phenotypic characters is a key component in organismal diversification; yet, the mechanisms underlying the emergence of such evolutionary novelties are largely unknown. Here we examine the origin of egg-spots, an evolutionary innovation of the most species-rich group of cichlids, the haplochromines, where these conspicuous male fin colour markings are involved in mating. Applying a combination of RNAseq, comparative genomics and functional experiments, we identify two novel pigmentation genes, fhl $a$ and fhl $2 b$, and show that especially the more rapidly evolving b-paralog is associated with egg-spot formation. We further find that egg-spot bearing haplochromines, but not other cichlids, feature a transposable element in the cis-regulatory region of fhl2b. Using transgenic zebrafish, we finally demonstrate that this region shows specific enhancer activities in iridophores, a type of pigment cells found in egg-spots, suggesting that a cis-regulatory change is causally linked to the gain of expression in egg-spot bearing haplochromines.

\footnotetext{
${ }^{1}$ Zoological Institute, University of Basel, Basel 4051, Switzerland. ${ }^{2}$ Institute of Neuroscience, University of Oregon, Eugene, Oregon 97403-1254, USA. ${ }^{3}$ Biozentrum, University of Basel, Basel 4056, Switzerland. †Present address: Institut de Génomique Fonctionnelle de Lyon, Ecole Normale Supérieure, CNRS UMR 5242, 46 Allée d'Italie, 69364 Lyon Cedex 07, France (M.E.S.); Evolutionary Ecology of Marine Fishes, GEOMAR Helmholtz Centre for Ocean Research Kiel, Düsternbrooker Weg 20, 24105 Kiel, Germany (B.S.M.). Correspondence and requests for materials should be addressed to M.E.S. (email: emilia.p.santos@gmail.com) or to W.S. (email: walter.salzburger@unibas.ch).
} 
$\mathrm{T}$ he de novo evolution of complex phenotypic traits poses a challenge to evolutionary biology ${ }^{1-5}$. While selection explains adaptation and speciation in an adequate manner ${ }^{6}$, it is more difficult to conceive how selection would trigger the origin of evolutionary novelties such as insect wings, feathers, tetrapod limbs, flowers, the mammalian placenta, beetle horns or butterfly eye-spots $1,4,5,7,8$. The emergence of evolutionary innovations, that is, lineage-restricted traits linked to qualitatively new functions, involves the origin of new developmental modules that are responsible for the identity of these novel characters ${ }^{4,5}$. Most of the available evidence suggests that new developmental programs emerge largely through cooption of pre-existing regulatory gene networks via changes in their regulation and deployment ('old genes playing new tricks ${ }^{5}$ ). Uncovering the mechanisms of how these developmental modules are co-opted or newly evolved is one of the primary goals of evo-devo research ${ }^{2,3,5,7,8}$.

Anal fin egg-spots are an evolutionary innovation in the so-called "haplochromines'9 (Fig. 1a and Supplementary Fig. 1), the most species-rich group of cichlid fishes, best known for their spectacular adaptive radiations in the East African lakes Victoria and Malawi ${ }^{10,11}$. Adult males of $\sim 1,500$ cichlid species feature this pigmentation trait in the form of conspicuously coloured circular markings $s^{9,11,12}$. Haplochromine egg-spots vary substantially in colour, shape, number and arrangement between species (Fig. 1b), and even within species a certain degree of variation is observed. In some species, also females show eggspots, which are then much less pronounced and colourful. The function of egg-spots has been implicated with the mating behaviour of the female-mouthbrooding haplochromines ${ }^{12,13}$. Immediately upon spawning, a haplochromine female gathers up her eggs into the mouth; the male then presents his egg-spots to which the female responds by snatching and bringing her mouth close to the male's genital opening; upon discharging sperm, the eggs become fertilized inside the female's mouth (Fig. 1c). The mother subsequently broods and carries her progeny in the oral cavities for several weeks after fertilization.

Here we are interested in the molecular basis of the anal fin egg-spots of haplochromine cichlids. The main advantages of the cichlid egg-spot system are that (i) the evolutionary innovation of interest emerged just a few million years ago and hence is recent compared with most other evolutionary novelties studied so far $9,10,14$; (ii) the phylogenetic context in which the novel trait evolved is known and living sister clades to the lineage featuring the novelty still exist ${ }^{9,15,16}$; and (iii) the genomes of two outgroup species lacking the trait and of three derived species featuring the trait are available. This allows us to study early events involved in the origin of an evolutionary innovation in an assemblage of phenotypically diverse, yet closely related and genetically similar species $^{14}$. Using RNAseq, we identify two novel candidate pigmentation genes, the a- and b-paralogs of the four and a half LIM domain protein 2 (fhl2) gene, and show that both genes, but especially the more rapidly evolving b-copy, are associated with the formation of egg-spots. We then find that egg-spot bearing haplochromines-but not an egg-spot-less ancestral haplochromine and not the representatives from more basal a

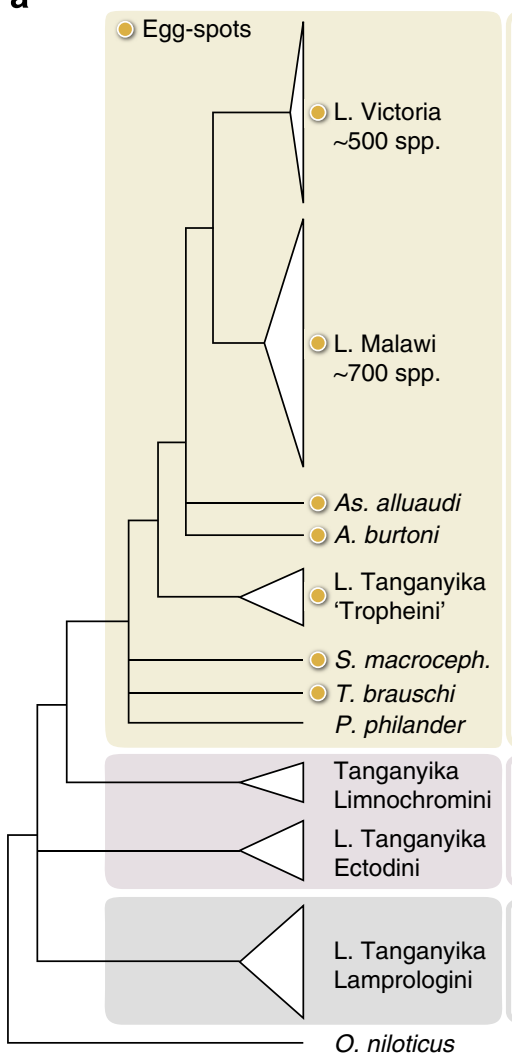

b
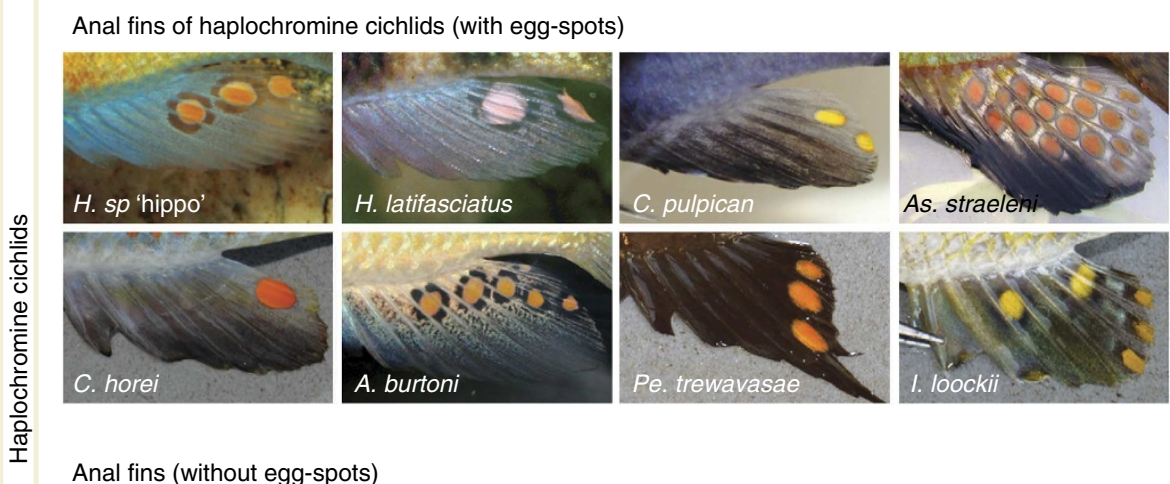

Anal fins (without egg-spots)
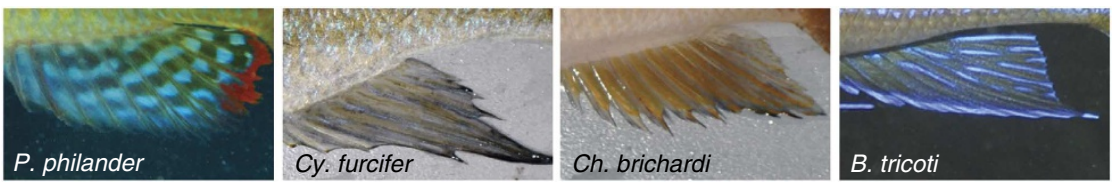

C

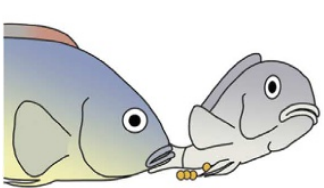

Egg laying

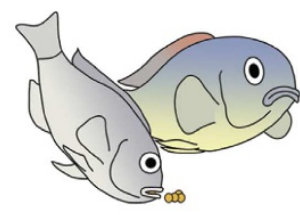

Egg uptake

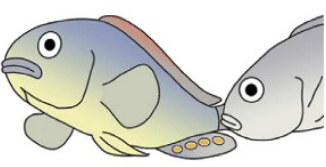

Fertilization

Figure 1 | The egg-spots of haplochromine cichlids. (a) Phylogeny of the East African cichlid fishes based on a new multimarker data set. The haplochromines are the most species-rich and derived group of cichlids in East Africa. One of the common features of haplochromines is the presence of egg-spots on the anal fin of males. Note that one of the ancestral lineages, represented here by $P$. philander, does not show this characteristic trait 9,33 . Substr-br, substrate brooders; mouthbr, mouthbrooders; spp.: species. (b) Examples of male anal fin patterns in East African cichlids. Haplochromine egg-spots (upper panel) vary in size, shape, number and colouration. Non-haplochromines and basal haplochromine P. philander (lower panel) do not show this trait. (c) A typical mating cycle of haplochromine cichlids. 
cichlid lineages-exhibit a transposable element insertion in close proximity to the transcription initiation site of $f h l 2 b$. A functional assay with transgenic zebrafish reveals that only a haplochromine-derived genetic construct featuring the SINE (short interspersed repetitive element) insertion drove expression in a special type of pigment cells, iridophores. Together, our data suggest that a cis-regulatory change (probably in the form of a SINE insertion) is responsible for the gain of expression of $f h l 2 b$ in iridophores, contributing to the evolution of egg-spots in haplochromine cichlids.

\section{Results}

fhl2 paralogs: novel candidates for egg-spot morphogenesis. As a first step, we performed an Illumina-based comparative transcriptomic experiment (RNAseq) between male (with egg-spots) and female (without egg-spots) anal fins in the haplochromine cichlid Astatotilapia burtoni. Two of the most differentially expressed genes according to RNAseq were the aand b-paralogs of fhl $2\left(\sim 4 \log _{2}\right.$-fold and $\sim 5 \log _{2}$-fold differences, respectively; see Supplementary Table 2). These paralogs result from the teleost genome duplication ${ }^{17}$ (Supplementary Fig. 2). The four and a half LIM domain protein 2 (Fhl2) is known as a transcriptional co-activator of the androgen receptor and the $W n t$-signalling pathway ${ }^{18,19}$; Fhl2 plays a role in cell-fate determination and pattern formation, in the organization of the cytoskeleton, in cell adhesion, cell motility and signal transduction; furthermore, it regulates the development of heart, bone and musculature in vertebrates ${ }^{20,21}$.

Expression of $\boldsymbol{f h l} 2 \boldsymbol{a}$ and $\boldsymbol{f h l} \boldsymbol{l} \boldsymbol{b}$ is egg-spot specific. To confirm the results obtained by RNAseq, we performed quantitative realtime PCR (qPCR) experiments (Fig. 2a), this time also comparing egg-spot versus non-egg-spot tissue within male anal fins. In addition, we tested another haplochromine species, Cynotilapia pulpican, with a different egg-spot arrangement to exclude positional effects of gene expression on the anal fin. In both species, the two duplicates of $f h l 2$ were overexpressed in egg-spots $(A$. burtoni: fhl2a: $t_{5}=10.77, P=0.0001$; fhl $2 b$ : $t_{5}=4.362, P=0.0073$; C. pulpican: fhl2a: $t_{4}=5.031, P=0.0073$; fhl $2 b$ : $t_{4}=9.154$, $P=0.0008)$. We then tested the expression of both fhl2 paralogs in the four main developmental stages of egg-spot formation in $A$. burtoni $^{22}$ and compared it with other candidate pigmentation genes (including the previously identified xanthophore marker csflra, the melanophore marker mitfa and the iridophore marker pnp4a). We found that the expression of both fhl 2 paralogs increases substantially throughout anal fin and egg-spot development, and both genes showed higher expression levels compared with the other pigmentation genes (Fig. 2b); fhl $2 b$ shows the highest increase in expression exactly when egg-spots begin to form. Furthermore, we corroborate that the expression a

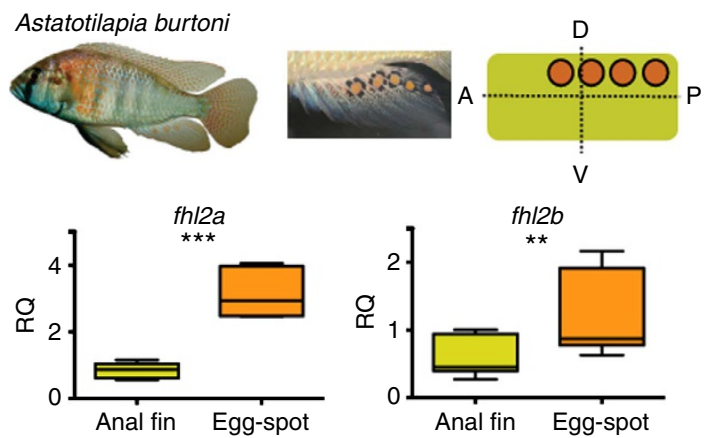

Cynotilapia pulpican
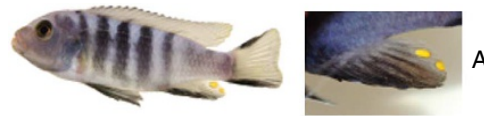

A
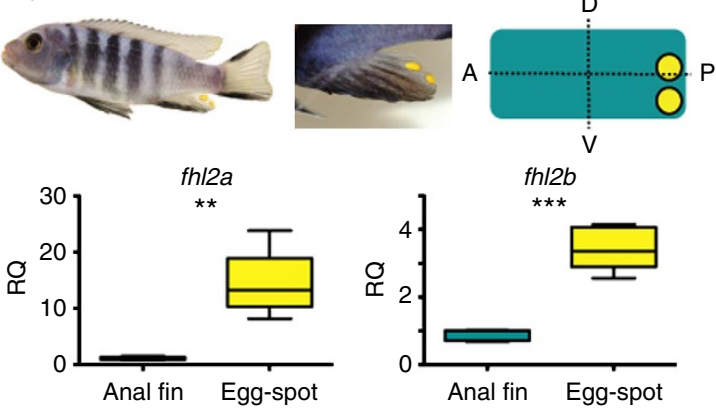

b
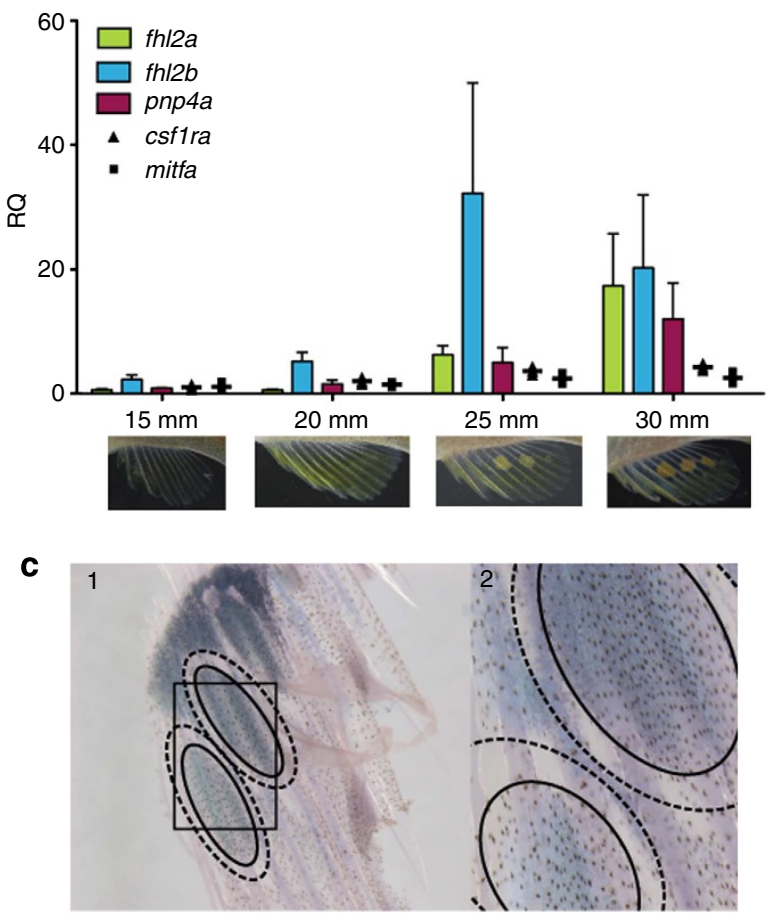

Figure $\mathbf{2}$ | The role of $\mathbf{f h l} \mathbf{2} \boldsymbol{a}$ and $\mathbf{f h l} \mathbf{2 b}$ in egg-spot formation. (a) qPCR experiments reveal that both genes are overexpressed in egg-spot compared with adjacent anal fin tissue in the haplochromine cichlids $A$. burtoni and $C$. pulpican ( ${ }^{\star \star} P<0.01$; ${ }^{\star \star \star} P<0.001 ; R Q$, relative quantity). Images of male fishes of the two species, their anal fins and a scheme showing the distribution of egg-spots are provided. (b) Expression profiles of fhl $2 a$ and fhl $2 b$ during the ontogenetic development of egg-spots in A. burtoni (note that egg-spots are absent in juveniles and only form when males become sexually mature; see ref. 22 for further details). The values on the $x$ axis represent fish standard length in millimetres (three replicates per developmental stage were used). The error bars represent the s.e.m. fhl $2 b$ shows the largest increase in expression overall and its expression profile mimics the formation of egg-spots. Three other pigmentation genes (pnp4a, csf1ra and mitfa) were included for comparative reasons. csflra and mitfa show a much smaller increase in gene expression during egg-spot development than fhl $2 a$ and especially fhl $2 b$, while pnp $4 a$ shows a constant increase in gene expression throughout the development of egg-spots. (c) RNA in situ hybridization experiments revealed that both fh/2 paralogs (results only shown for fhl2b) are primarily expressed in the colourful inner circle of haplochromine egg-spots (defined by the solid line) and not in the transparent outer ring (defined by the dashed line). Expression was also observed in the proximal fin region, which also contains pigment cells. Panel 2 is a close-up from the region defined by the square in panel 1. 
domain of both fhl2a and fhl2b matches the conspicuously coloured inner circle of egg-spots with RNA in situ hybridization (see Fig. 2c for results on fhl2b).

$f h l 2 a$ and $f h l 2 b$ evolved under purifying selection. In general, phenotypic differences can arise via mutations affecting the function of proteins or via changes in gene regulation ${ }^{5}$. Therefore, we examined coding sequence evolution in the two fhl2 paralogs to test for positive selection and potential change of function in a phylogenetically representative set of 26 East African cichlids. We found that the two fhl 2 genes are highly conserved in cichlids, with few amino-acid differences between species and an average genetic divergence $(0.4 \%$ in $f h l 2 a$ and $0.7 \%$ in $f h l 2 b)$ that lies below the transcriptome-wide average of $0.95 \%$ (ref. 23). None of the observed amino-acid changes was correlated with the eggspot phenotype (Supplementary Table 7).

Greater functional specialization of $\boldsymbol{f h l} \mathbf{b} \boldsymbol{b}$ in haplochromines. Usually, after a gene duplication event, the duplicates go through a period of relaxed selection, during which one of the two copies can diversify and acquire new functions ${ }^{24}$. We found that the b-copy of fhl2 shows an elevated rate of molecular evolution compared with its paralog $(f h l 2 a)$, which more closely resembles the ancestral sequence (Fig. 3a). An additional series of qPCR experiments in 12 tissues revealed that, in cichlids, fhl2a is primarily expressed in heart, bony structures and muscles, whereas fhl $2 b$ is highly expressed in the eye, and further in skin and the egg-spots of haplochromines (Fig. 3b,c). This is different to the gene expression profiles in medaka, where both duplicates are highly expressed in heart, skin and eye tissues; and in zebrafish, where the two paralogs are primarily expressed in heart, eye and (pharyngeal) jaw tissues, with fhl2a showing rather low levels of gene expression (Supplementary Figs 3 and 4 ). When compared with the other teleost fishes examined here, our results suggest that the haplochromine fhl2a retained most of the previously described functions, whereas the more rapidly evolving fhl $2 b$ obtained new expression patterns. Together, the gene expression profile and the pattern of sequence evolution make fhl $2 b$ a prime candidate gene for the morphogenesis of haplochromine egg-spots.

fhl2b shows an AFC-SINE insertion in species with egg-spot. Since there were no changes in the coding regions of fhl $a$ and fhl $2 b$ that are specific to the egg-spot bearing haplochromines, we shifted our focus towards the analysis of putative regulatory elements, exploring the recently available genomes of five East African cichlids (including the egg-spot bearing haplochromines A. burtoni, Pu. nyererei, Metriaclima zebra and the egg-spot-less non-haplochromines Neolamprologus brichardi and Oreochromis niloticus). The non-coding region of fhl2a shows homology with other teleosts (Oryzias latipes, Takifugu rubripes, Tetraodon nigroviridis and Gasterosteus aculeatus) and we identified four conserved non-coding elements (CNEs) in all species examined (Supplementary Fig. 5a). These CNEs might thus represent conserved regulatory regions responsible for ancestral conserved functions of fhl $2 a$ in teleosts. We might be missing cichlid-specific regulatory regions in important upstream regions although, as our capacity to detect lineage-specific enhancers is limited owing to the small sample size for each lineage and the high background conservation level present in cichlids.

Concerning fhl $2 b$, we did not find any CNE that is shared by cichlids and other teleosts (Supplementary Fig. 5b). Strikingly, however, we found a major difference that is shared by the three egg-spot bearing haplochromines: the presence of a transposable element upstream of $f h l 2 b$. Specifically, we identified a SINE belonging to the cichlid-specific AFC-SINEs (African cichlid family of SINEs ${ }^{25}$ ), which inserted $\sim 800$-bp upstream of the transcriptional start site of fhl2b (Supplementary Fig. 6). To confirm that this insertion is associated with the egg-spot phenotype, we sequenced the upstream region of fhl2b in 19 cichlid species. The insertion was indeed present in nine additional, egg-spot bearing haplochromine species, yet absent in all 10 non-haplochromines examined (Supplementary Table 8). Importantly, we found that one haplochromine species lacks the AFC-SINE element, namely $P$. philander. This species belongs to one of the basal lineage of haplochromines (Fig. 1a), which is characterized by the absence of egg-spots (Fig. 1b). This suggests that the AFC-SINE upstream of fhl $2 b$ is not characteristic to the entire haplochromine clade, but to those that feature egg-spots, thus linking the SINE insertion to the origin of this evolutionary innovation.

Haplochromine $f h l 2 b$ regulatory region drives iridophore expression. A long-standing hypothesis proposes that ubiquitous genomic repeat elements are potential regulators of transcription, and could thereby generate evolutionary variations and novelties $^{26,27}$. SINEs are known for their capability of 'transcriptional rewiring, that is, to change the expression patterns of genes by bringing along new regulatory sequences when inserted in close proximity to a gene's transcriptional initiation site ${ }^{7,28}$. In order to test whether the insertion of an AFC-SINE close to fhl $2 b$ functions as an enhancer of gene expression, we aimed for a functional experiment. We were particularly interested to find out whether there were changes in enhancer activity between AFCSINE-positive haplochromines and other cichlids lacking both the insertion and the egg-spot phenotype. To this end, we designed reporter constructs containing the upstream region of $f h l 2 b$ ( $\sim 2 \mathrm{~kb}$ upstream to intron 1 ) of three cichlid species linked to the coding region of green fluorescent protein (GFP), and injected these constructs into zebrafish (Danio rerio) embryos to generate transgenic lines. We switched to the zebrafish system here, as no functioning transgenesis was available for haplochromine cichlids at the time the study was performed (owing to the small number of eggs per clutch associated with the characteristic femalemouthbrooding behaviour). The three constructs were derived from A. burtoni (haplochromine with egg-spots, AFC-SINE ${ }^{+}$), P. philander (haplochromine without egg-spots, AFC-SINE ${ }^{-}$) and N. sexfasciatus (lamprologine, $\mathrm{AFC}^{-\mathrm{SINE}^{-}}{ }^{-}$), respectively (Fig. 4a).

We were able to produce stable transgenic zebrafish lines for each of the three constructs to examine the expression of GFP. Importantly, we found striking differences in expression between the A. burtoni construct and the two constructs lacking the AFC-

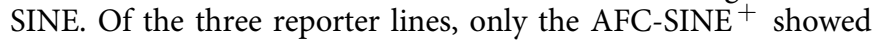
GFP expression in iridophores, a silvery-reflective type of pigment cells (Fig. 4b,c and Supplementary Fig. 7). This experiment demonstrates the presence of novel enhancer activities in the regulatory region of $f h l 2 b$ in derived haplochromines and strongly suggests that these came along with the SINE insertion.

Iridophores and egg-spot development. The egg-spot phenotype has previously been associated with pigment cells containing pteridines (xanthophores) ${ }^{16,22}$, whereas our new results indicate an auxiliary role of iridophores in egg-spot formation. We thus re-evaluated the adult egg-spot phenotype by removing the pteridine pigments of the xanthophores (Fig. 4e). We indeed found that A. burtoni egg-spots show a high density of iridophores, which is further corroborated by the increase in gene expression of the iridophore marker pnp4a during egg-spot 


\section{a}

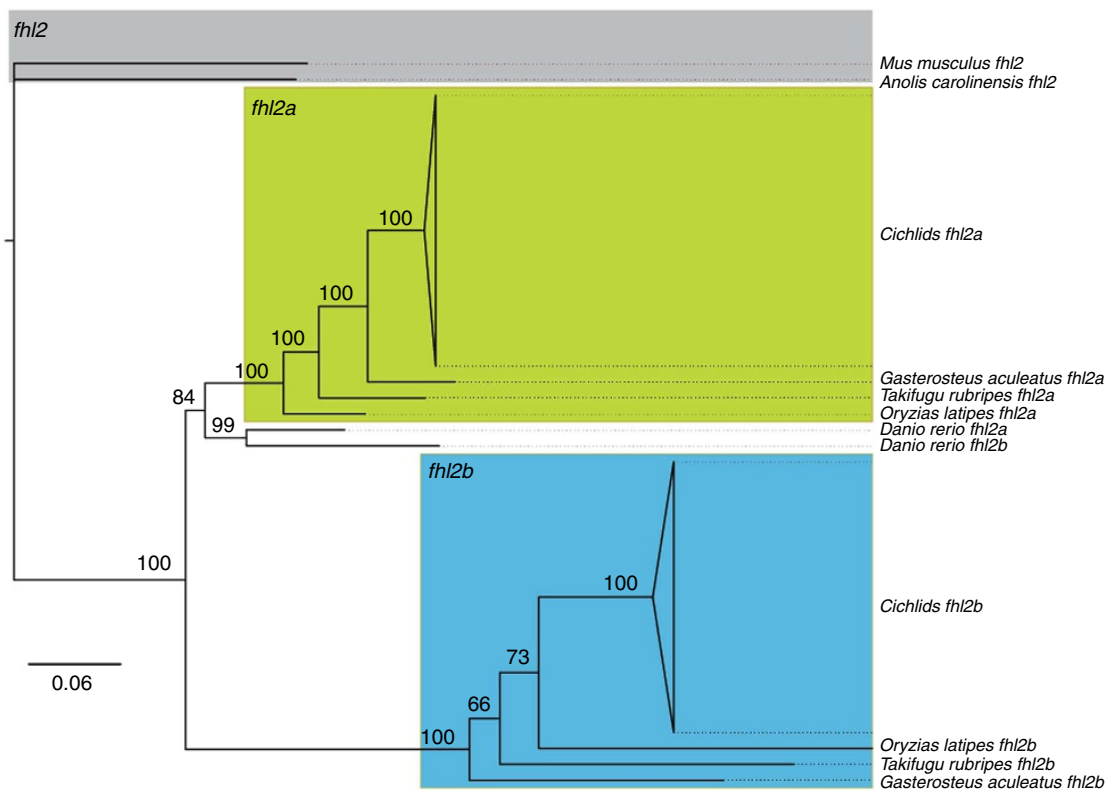

b

Cynotilapia pulpican
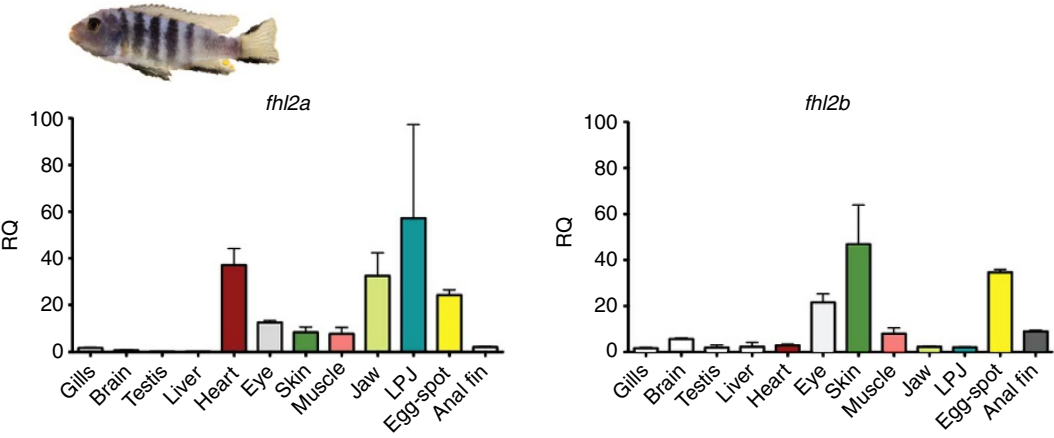

C
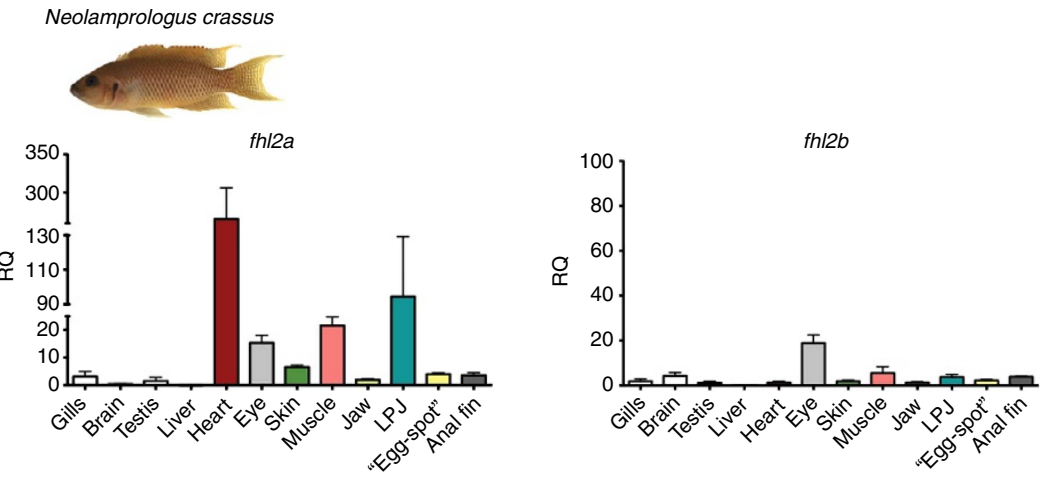

Figure 3 | Gene tree of the two fhl2 paralogs and expression profiling in East African cichlid fishes. (a) Bayesian inference phylogeny of the orthology and paralogy relationships between cichlids, other teleosts (O. latipes, D. rerio, Ta. rubripes and G. aculeatus) and tetrapods (Anolis carolinensis and Mus musculus) fhl 2 sequences. This gene tree is important for generating functional hypotheses about both duplicates, and to infer the ancestral state of the fhl 2 gene before duplication. Our phylogeny indicates that $f h / 2 a$ is more similar to the ancestral state, while fhl $2 b$ is apparently evolving faster in teleosts. Values at the tree nodes represent posterior probabilities. In Supplementary Fig. 2, we present a synteny analysis supporting the origin of teleost fh/2 duplicates in the teleost genome duplication. (b) Relative quantity (RQ) of fhl2a and fhl2b gene expression in 12 tissues (three replicates per tissue) in $C$. pulpican, an egg-spot bearing haplochromine from Lake Malawi. The error bars represent the s.e.m. (c) RQ of fhl $2 a$ and fhl $2 b$ gene expression in 12 tissues in $N$. crassus, a substrate spawning lamprologine that has no egg-spots. In both species, gill tissue was used as reference; in N. crassus, 'egg-spots' corresponds to the fin region where haplochromines would show the egg-spot trait. In C. pulpican (b), fhl2a is highly expressed in heart, in pigmented tissues (eye, skin and egg-spot) and in craniofacial traits (oral jaw and lower pharyngeal jaw); fhl $2 b$ is mainly expressed in the pigmented tissues. $N$. crassus (c) shows a similar expression patterns for fhl $2 a$ and $f h l 2 b$, with the difference that fhl2a does not show high expression levels in jaw tissues, and fhl $2 b$ is not highly expressed in skin and fin tissue. These results suggest that fhl $2 b$ shows a higher functional specialization, and that it might be involved in the morphogenesis of sexually dimorphic traits such as pigmented traits including egg-spots. LPJ, lower pharyngeal jaw bone. 


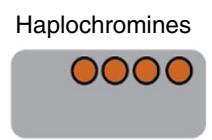

Egg-spots

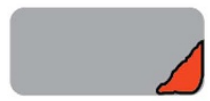

Non-haplochromines

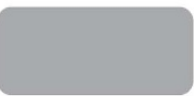

$5^{\prime}$

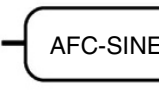

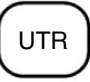

Intron 1

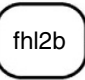

$-3^{\prime}$
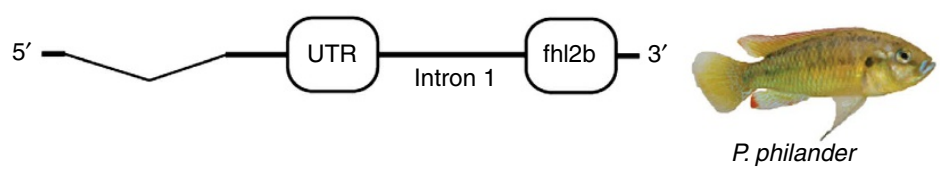

$5^{\prime}$
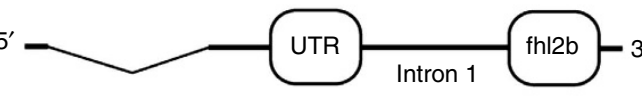

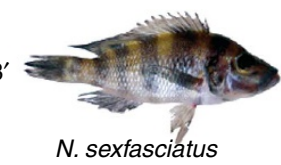

b

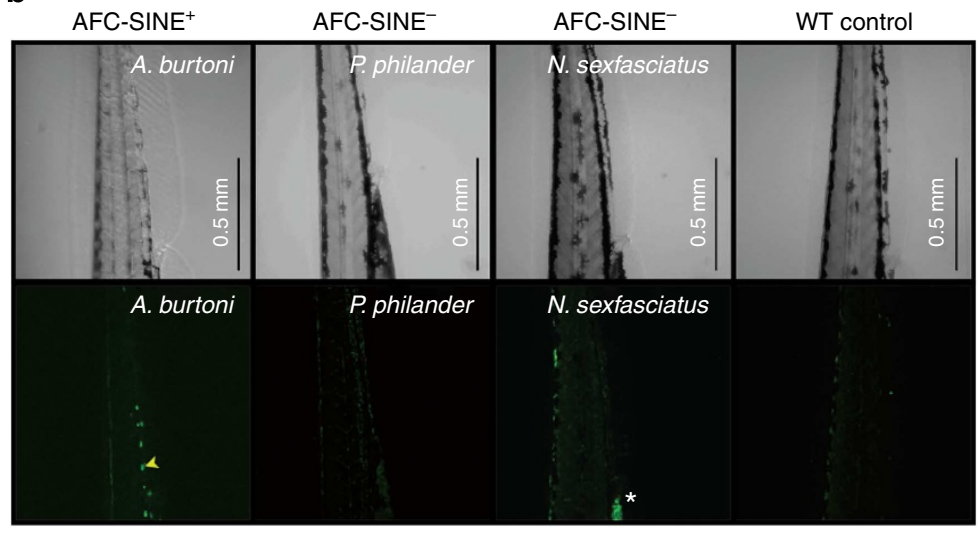

c

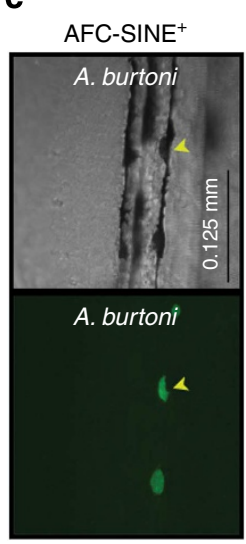

d AFC-SINE $^{+}$

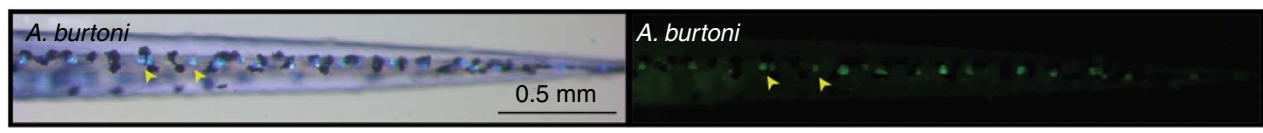

e

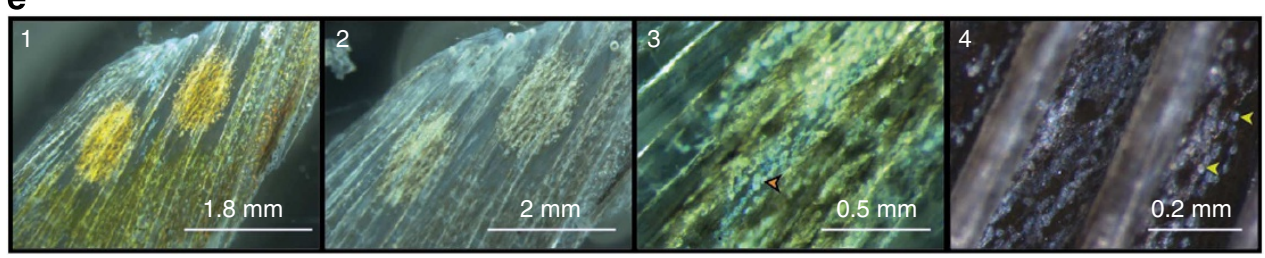

Figure 4 | The molecular basis of egg-spot formation. (a) The egg-spot bearing haplochromines feature an AFC-SINE insertion in close proximity to the transcriptional start site of fhl2b, which is absent in the ancestral and egg-spot-less genus Pseudocrenilabrus and in all non-haplochromines. The sequences from the three species shown here were the ones used to engineer the reporter constructs, where the fhl $2 b$ coding sequence was substituted by GFP. (b) In transgenic zebrafish, only the AFC-SINE ${ }^{+}$construct showed GFP expression in the iridophores, a type of pigment cells (one of them is indicated by a yellow arrow). The upper panel depicts bright-field images of 3-day-old zebrafish embryo trunks; the lower panel shows the respective embryos under ultraviolet light. The green signal in the AFC-SINE negative N. sexfasciatus line (marked with an asterisk) is auto-fluorescence from the yolk extension. (c) Higher magnification image from A. burtoni AFC-SINE ${ }^{+}$reporter construct driving GFP expression in the iridophores. Orientation in b,c: bottom: anterior, top: posterior. (d) Top-down view of a trunk of a 3-day-old AFC-SINE-positive zebrafish embryo. The left panel depicts a bright-field image where the iridophores of the dorsal stripe are illuminated by the incident light (yellow arrows). The right panel depicts GFP expression of the same embryo. The GFP signal co-localizes with iridophores. (e) Cellular basis of egg-spots: this series of images shows that egg-spots are made up of xanthophores, iridophores and scattered melanophores. Image 1 shows an A. burtoni fin with two egg-spots. Image 2 shows the same fin without pteridine pigments (xanthophores are not visible anymore). Images 3 and 4 are higher magnification images of the egg-spots without pteridine under slightly different light conditions confirming that egg-spots have a high density of iridophores (examples of this cell type are highlighted with arrows). UTR, untranslated region.

formation (Fig. 2b). With the exception of the proximal region of the anal fin, the number of iridophores is greatly reduced in the fin tissue surrounding egg-spots (Supplementary Fig. 8a). Interestingly, this proximal region is the only area of the anal fin besides the egg-spots where we observed fhl2 expression with
RNA in situ hybridization (see Fig. 2c for $f h l 2 b$ ), once more linking fhl2 expression with iridophores (and less so with xanthophores, which are very rare in this region). In the nonhaplochromine $N$. crassus, which features a yellow anal fin pattern containing xanthophores, we did not find iridophores in 
the xanthophore-rich region (Supplementary Fig. 9), suggesting that the xanthophore/iridophore pattern is unique to haplochromine egg-spots. Importantly, we also observed that iridophores appear early in the newly forming egg-spot of haplochromines, that is, before the first xanthophores start to aggregate (Supplementary Fig. 8b).

In zebrafish, stripe development is initiated by iridophores, which serve as morphological landmarks for stripe orientation in that they attract further pigment cells such as xanthophores by expressing the csf 1 ligand gene ${ }^{29,30}$. Interestingly, it has previously been shown that a gene encoding a Csf1 receptor known for its role in xanthophore development in zebrafish, csflra, is expressed in haplochromine egg-spots ${ }^{16}$. We thus examined the expression of the ligand $c s f 1 b$ and show that its relative level of gene expression doubles during egg-spot development, and that this increase coincides with the emergence of the phenotype (Supplementary Fig. 10). This leads us to suggest that a similar pigment cell type interaction mechanism might be involved in egg-spot patterning as the one described for zebrafish ${ }^{29,30}$. The specific mode of action of fin patterning in haplochromine cichlids, and how Fhl2b interacts with the Csf1/Csf1r system, remains to be studied in the future.

Contribution of $f h l 2 a$ in egg-spot formation. The role of the more conserved and functionally constrained a-paralog of fhl2 in egg-spot development cannot be dismissed. Its temporally shifted increase in gene expression compared with $f h l 2 b$ (Fig. 2b) suggests that fhl2a most likely acts as a more downstream factor involved in pigment pattern formation. We were nevertheless interested in uncovering the regulatory region responsive for this expression pattern. The first intron of fhl2a shows two CNEs that are common across percomorph fish (Supplementary Fig. 5). Using the same strategy as described above, we generated a transgenic zebrafish line containing exon 1 and intron 1 of A. burtoni linked to GFP. This construct drove expression in heart in zebrafish embryos, which is consistent with the reported function of fhl2a in tetrapods ${ }^{20}$, whereas there was no indication of a pigment cell related function for this reporter construct (Supplementary Fig. 7e). An alignment between the genomic regions of the two fhl 2 paralogs shows that there were no CNEs in common and generally very little homology between them, suggesting that the regulation of the expression of fhl2a in eggspots might proceed in a different way (Supplementary Fig. 11).

\section{Discussion}

In this study, we were interested in the genetic and developmental basis of egg-spots, an evolutionary innovation of the most species-rich group of cichlids, the haplochromines, where these conspicuous colour markings on the anal fins of males play an important role in mating ${ }^{11-13}$ (Fig. 1).

We first performed a comparative RNAseq experiment that led to the identification of two novel candidate pigmentation genes, the a- and b-paralogs of the four and a half LIM domain protein 2 (fhl2) gene. We then confirmed, with qPCR and RNA in situ hybridization, that the expression domain of both duplicates indeed matches the conspicuously coloured inner circle of eggspots (Fig. 2). Especially the more rapidly evolving b-copy of fhl2 emerged as strong candidate gene for egg-spot development, as its expression profile mimics the formation of egg-spots (Figs $2 \mathrm{~b}$ and 3). Interestingly, we found that the egg-spot bearing haplochromines, but not other cichlids, feature a transposable element in the cis-regulatory region of fhl $b$. Finally, making use of transgenic zebrafish, we could show that a cis-regulatory change in fhl $2 b$ in the ancestor of the egg-spot bearing haplochromine cichlids (most likely in the form of the AFC-SINE insertion) resulted in a gain of expression in iridophores, a special type of pigment cells found in egg-spots (Fig. 4). This in turn might have led to changes in iridophore cell behaviour and to novel interactions with pigmentation genes (csflb, csflra and pnp4a), thereby contributing to the formation of egg-spots on male anal fins. The specific mode of action of the SINE insertion, and how the fhl $2 b$ locus interacts with these other pigmentation genes remains elusive at present. Addressing these questions would require functional studies in haplochromines, which are, however, hampered by the specific mechanisms involved in the trait complex of interest (mouthbrooding makes it notoriously difficult to obtain enough eggs-in a controlled manner-to make such experiments feasible).

Our results are also suggestive of an important role of the a-copy of fhl2 in cichlid evolution. With our qPCR experiments, we provide strong evidence that fhl2a is involved in jaw tissue in zebrafish (Supplementary Fig. 3) and, importantly, in the pharyngeal jaw apparatus of cichlids (Fig. 3b,c), another putative evolutionary innovation of this group. The pharyngeal jaw apparatus is a second set of jaws in the pharynx of cichlids that is functionally decoupled from the oral jaws and primarily used to process food ${ }^{11,12,15}$. Interestingly, fhl2a has previously been implicated in the evolution of fleshy lips in cichlids ${ }^{31}$, which is yet another ecologically relevant trait. From a developmental perspective, the main tissues underlying these traits-the craniofacial cartilage (the jaw apparatus) and pigment cells (eggspots)-have the same origin, the neural crest, which itself is considered an evolutionary key innovation of vertebrates ${ }^{32}$. It thus seems that the function of fhl 2 in cichlids may have been split into (a) an ecologically important, that is, naturally selected, scope of duties, and (b) a role in colouration and pigmentation more likely to be targeted by sexual selection.

Taken together, our study permits us to propose the following hypothesis for the origin of cichlid egg-spots: In one of the early, already female-mouthbrooding, haplochromines the insertion of a transposable element of the AFC-SINE family in the cisregulatory region of $f h l 2 b$, and its associated recruitment to the iridophore pigment cell pathway, mediated the evolution of eggspots on the anal fins-possibly from the so-called perfleckmuster common to many cichlids ${ }^{16}$. The conspicuous anal fin spots were fancied by haplochromine females, which-just like many other cichlids and also the ancestral and egg-spot-less haplochromine genus Pseudocrenilabrus - have an innate bias for yellow/orange/ red spots that resemble carotenoid-rich prey items ${ }^{33}$, leading to the fixation of the novel trait. In today's haplochromines, eggspots seem to have a much broader range of functions related to sexual selection ${ }^{34}$.

Most of the currently studied evolutionary innovations comprise relatively ancient traits (for example, flowers, feathers, tetrapod limb, insect wings and mammalian placenta), thereby making it difficult to scrutinize their genetic and developmental basis. Here we explored a recently evolved novelty, the anal fin egg-spots of male haplochromine cichlids. We uncovered a regulatory change in close proximity to the transcriptional start site of a novel iridophore gene that likely contributes to the molecular basis of the origin of egg-spots in the most rapidly diversifying clade of vertebrates. This, once more, illustrates the importance of changes in cis-regulatory regions in morphological evolution ${ }^{2}$

\section{Methods}

Samples. Laboratory strains of A. burtoni, C. pulpican, Astatoreochromis alluaudi, $P u$. nyererei, Labidochromis caeruleus, Pseudotropheus elegans and N. crassus were kept at the University of Basel (Switzerland) under standard conditions (12 h light/ $12 \mathrm{~h}$ dark; $\left.26^{\circ} \mathrm{C}, \mathrm{pH} 7\right)$. Before dissection, all specimens were euthanized with MS 222 (Sigma-Aldrich, USA) following an approved procedure (permit no. 2317 
issued by the cantonal veterinary office Basel). Individuals of all other specimens were collected in the southern region of Lake Tanganyika (Zambia) under the permission of the Lake Tanganyika Unit, Department of Fisheries, Republic of Zambia, and processed in the field following our standard operating procedure ${ }^{15}$. Tissues for RNA extraction were stored in RNAlater (Ambion, USA), and tissues for genomic DNA extraction were stored in ethanol and shipped to the University of Basel.

RNA and DNA extractions. Isolation of RNA was performed according to the TRIzol protocol (Invitrogen, USA) after incubating the dissected tissues in $750 \mu \mathrm{l}$ of TRIzol at $4{ }^{\circ} \mathrm{C}$ overnight or, alternatively, for $8-16 \mathrm{~h}$ (in order to increase the RNA yield after long-term storage). The tissues were then homogenized with a BeadBeater (FastPrep-24; MP Biomedicals, France). Subsequent DNase treatment was performed with DNA-Free kit (Ambion). RNA quantity and quality was determined with a NanoDrop 1000 spectrophotometer (Thermo Scientific, USA). cDNA was produced using the High Capacity RNA-to-cDNA kit (Applied Biosystems, USA). Genomic DNA was extracted using a high salt extraction method (modified from ref. 35).

Phylogenetic analyses. DNA extraction of 18 specimens of East African cichlid fishes was conducted as described above. For the amplification of nine nuclear markers (rag, gapdhs, s7, bmp4, ednrb1, mitfa, tyr, hag and csfr1), we used the primer sets published in ref. 36. The sequences of M. zebra, O. niloticus and N. brichardi were extracted from the respective genome assemblies (http://www. broadinstitute.org/models/tilapia). The data for Astatoreochromis alluaudi,

Thoracochromis brauschi and Serranochromis macrocephalus were collected with Sanger sequencing following the method described in ref. 36, all other data were generated by amplicon sequencing with 454 GS FLX system at Microsynth, Switzerland, following the manufacturer's protocols ${ }^{37,38}$. Sequences were quality filtered using PRINSEQ (length: $150 \mathrm{bp}$ minimum; low quality: mean $\geq 15$; read

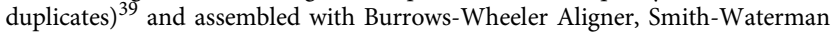
alignment (BWA-SW) followed by visual inspection and consensus sequence generation in Geneious 6.1.6 (ref. 40). As a tenth marker, we included mitochondrial NADH dehydrogenase subunit 2 (ND2) sequences available on GenBank (see Supplementary Table 1 for accession numbers). Since the ednrb1 gene sequence is not available in the $N$. brichardi genome assembly, we used the gene sequence from its sister species, $N$. pulcher, instead.

Sequences were aligned with MAFFT ${ }^{41}$ and the most appropriate substitution model of molecular evolution for each marker was determined with JMODELTEST v2.1.3 (ref. 42) and BIC ${ }^{43}$. The partitioned data set $(5,051 \mathrm{bp})$ was then subjected to phylogenetic analyses in MRBAYES v3.2.1 (ref. 44) and GARLI v2.0 (ref. 45). MRBAYES was run for 10,000,000 generations with two runs and four chains in parallel and a burn-in of $25 \%$, GARLI was run 50 times followed by a bootstrap analysis with 500 replicates. SUMTREES v3.3.1 of the DENDROPY package v3.12.0 (ref. 46) was used to summarize over the replicates and to map bootstrap values to the ML topology.

Differential gene expression analysis using RNAseq. We used a transcriptomic approach (RNAseq) to identify genes differentially expressed between male and female anal fins of A. burtoni. Library construction and sequencing of RNA extracted from three male and three female anal fins (at the developmental stage of $30 \mathrm{~mm}$; Fig. 2) was performed at the Department of Biosystems Science and Engineering, University of Basel and ETH Zurich. The samples were sequenced on an Illumina Genome Analyzer IIx. Each sample was sequenced in one lane and with a read length of $76 \mathrm{bp}$.

The reads were then aligned to an embryonic $A$. burtoni reference transcriptome assembled by Broad Institute (http://www.broadinstitute.org/ models/tilapia). This transcriptome is not annotated and each transcript has a nomenclature where the first term codes for the parent contig and the third term codes for alternatively spliced transcripts (CompX_cX_seqX). The reference transcriptome was indexed using NOVOINDEX (www.novocraft.com) with default parameters. Using NOVOALIGN (www.novocraft.com), the RNAseq reads were mapped against the reference transcriptome with a maximum alignment $(t)$ score of 30, a minimum of good-quality base pair per read (l) of 25 and a successive trimming factor (s) of 5 . Reads that did not match these criteria were discarded. Since the reference transcriptome has multiple transcripts/isoforms belonging to the same gene, all read alignment locations were reported (rALL). The mapping results were reported (o) in SAM format. The output SAM file was then transformed into BAM format, sorted, indexed and converted to count files (number of reads per transcript) using SAMTOOLS version 0.1.18 (ref. 47). The count files were subsequently concatenated into a single data set - count table - and analysed with the R package EDGER ${ }^{48}$ in order to test for significant differences in gene expression between male and female anal fins. The 10 most differentially expressed transcripts were identified by BLASTx ${ }^{49}$ against GenBank's nonredundant database (Supplementary Table 2).

We selected two genes out of this list for in-depth analyses-fhl2a and fhl $2 b$-for the following three reasons: (i) fhl $2 b$ was the gene showing the highest difference in expression between male and female anal fins; (ii) the difference in gene expression in its paralog, fhl2a, was also significantly high; and (iii) the functional repertoire of the Fhl2 protein family indicates that these might be strong candidates for the morphogenesis of a secondary male colour trait.

Differential gene expression analysis using qPCR. The expression patterns of $f h l 2 a$ and $f h l 2 b$ were further characterized by means of qPCR in three species, A. burtoni, C. pulpican and N. crassus. The comparative cycle threshold method ${ }^{50}$ was used to calculate differences in expression between the different samples using the ribosomal protein $\mathrm{L} 7(\mathrm{rpl} / 7)$ and the ribosomal protein SA3 ( $r p s a 3)$ as endogenous controls. All reactions had a final cDNA concentration of $1 \mathrm{ng} \mu \mathrm{l}^{-1}$ and a primer concentration of $200 \mathrm{mM}$. The reactions were run on a StepOnePlusTM Real-Time PCR system (Applied Biosystems) using the SYBR Green master mix (Roche, Switzerland) with an annealing temperature of $58^{\circ} \mathrm{C}$ and following the manufacturer's protocols. Primers were designed with the software GenScript Real-Time PCR (Taqman) Primer Design available at https:// www.genscript.com/ssl-bin/app/primer. All primers were designed to span over exons to avoid gDNA contamination (see Supplementary Table 3 for details). Primer efficiencies of the experimental primers ( $f h l 2 a$ and $f h l 2 b)$ were comparable to the efficiency of the endogenous controls rpl7 and rpsa3.

We conducted the following experiments: qPCR experiment 1: Egg-spots were separated from the anal fin tissue in six male A. burtoni and five male C. pulpican. Relative quantity values were calculated for each sample, and the differential expression between anal fin (reference) and egg-spot tissue was analysed with a paired $t$-test using GraphPad Prism version 5.0a for Mac OS X (www.graphpad.com). qPCR experiment 2: fhl2a, fhl2b, csflra, mitfa, pnp4a and $c s f 1 b$ expression was measured in RNA extracted from A. burtoni fins at four different developmental stages ${ }^{22}$. Here, csflra was included as xanthophore marker ${ }^{16}$, mitfa and $p n p 4 a$ as melanophore and iridophore markers ${ }^{51}$, respectively, and $c s f 1 b$ because of its role in pigment pattern organization in zebrafish ${ }^{29,30}$. We used three biological replicates for each developmental stage, and each replicate consisted of a sample pool of three fins, except for the youngest stage at $15 \mathrm{~mm}$, where we pooled five fins. The first developmental stage was used as reference tissue. QPCR experiment 3: fhl2a and $f h l 2 b$ expression was measured in RNA extracted from different tissues from three males from C. pulpican and N. crassus (gills, liver, testis, brain, heart, eye, skin, muscle, oral jaw, pharyngeal jaw and eggspot). Although $N$. crassus does not have egg-spots, we separated its anal fin into an area corresponding to egg-spots in haplochromines and a section corresponding to anal fin tissue (the 'egg-spot' region was defined according to the egg-spot positioning in A. burtoni). Expression was compared among tissues for each species using gills as reference tissue. The same experiment was performed for $D$. rerio and $O$. latipes (two teleost outgroups), using efla and rpl13a (ref. 52), as well as $r p l 7$ and 18sRNA (ref. 53) as endogenous controls, respectively.

Cloning of fhl2a and fhl2b and RNA in situ hybridization. A. burtoni fhl2a and fhl $2 b$ coding fragments were amplified by PCR (for primer information, see Supplementary Table 3) using Phusion Master Mix with High Fidelity buffer (New England BioLabs, USA) following the manufacturer's guidelines. These fragments were cloned into pCR4-TOPO TA vector using the TOPO TA cloning kit (Invitrogen). Plasmid extractions were done with GenElute Plasmid Miniprep Kit (Sigma-Aldrich). RNA probes were synthetized with the DIG RNA labelling kit (SP6/T7) (Roche). The insertion and direction of the fragments was confirmed by Sanger sequencing using M13 primers (available with the cloning kit) and BigDye terminator reaction chemistry (Applied Biosystems) on an AB3130xl Genetic Analyzer (Applied Biosystems). In situ hybridization was performed in 12 fins from A. burtoni males, six for $f h l 2 a$ and six for fhl $2 b$. The protocol was executed as described in ref. 16, except for an intermediate proteinase $K$ treatment $(20 \mathrm{~min}$ at a final concentration of $\left.15 \mu \mathrm{g} \mathrm{ml}^{-1}\right)$ and for the hybridization temperature $\left(65^{\circ} \mathrm{C}\right)$.

Synteny analysis of teleost fhl/2 paralogs. The Synteny Database (http://syntenydb.uoregon.edu ${ }^{54}$ ) was used to generate dotplots of the human FHL2 gene (ENSG00000115641) region on chromosome Hsa2 and the genomes of medaka (Supplementary Fig. 2a) and zebrafish (Supplementary Fig. 2b). Double-conserved synteny between the human FHL2 gene and the fhl $2 a$ and $f h l 2 b$ paralogons in teleost genomes provide evidence that the teleost $f h l 2$ paralogs were generated during the teleost genome duplication.

fhl2a/fhl2b coding region sequencing and analysis. We then used cDNA pools extracted from anal fin tissue to amplify and sequence the coding region of fhl2a and $f h l 2 b$ in a phylogenetically representative set of 26 cichlid species (21 Tanganyikan species, three species from Lake Malawi and two species from the Lake Victoria basin). This taxon sampling included 14 species belonging to the haplochromines and 12 species belonging to other East African cichlid tribes not featuring the egg-spot trait (Supplementary Table 4). fhl2a and fhl2b coding regions were fully sequenced (from start to stop codon) in five individuals per species in order to evaluate the rate of molecular evolution among cichlids. For PCR amplification, we used Phusion Master Mix and cichlid-specific primers (for primer information, see Supplementary Table 3) designed with Primer3 (ref. 55). PCR products were visualized with electrophoresis in a $1.5 \%$ agarose gel using GelRed (Biotium, USA). In cases where multiple bands were present, we purified the correct size fragment from the gel using the GenElute Gel Extraction Kit (Sigma- 
Aldrich). PCR products were enzymatically cleaned with ExoSAP-IT (Affymetrix, USA) and sequenced with BigDye 3.1 Ready reaction mix (Applied Biosystems) after BigDye XTerminator purificaton (Applied Biosystems) - on an AB3130xl Genetic Analyzer. Sequences were corrected, trimmed and aligned manually in CODONCODE ALIGNER (CodonCode Corporation).

fhl2 phylogenetic analysis. fhl2a and $f h l 2 b$ sequences from non-cichlid teleosts and fhl 2 sequences from tetrapods were retrieved from ENSEMBL ${ }^{56}$ (species names, gene names and accession numbers are available in Supplementary Table 5). We then constructed gene trees based on these sequences and on a subset of the cichlid sequences obtained in the previous step (information available in Supplementary Table 4) in order to confirm the orthologous and paralogous relationships of both duplicates. Sequences were aligned with cLustalw2 (ref. 57) using default parameters. The most appropriate model of sequence evolution was determined with JMODELTEST as described above. Phylogenetic analyses were performed with MRBAYES (1 million generations; $25 \%$ burn-in).

Tests for positive selection in fhl2a and fhl2b. Using PAUP ${ }^{\star} 4.0 \mathrm{~b} 10$ (ref. 58), we first compiled a maximum likelihood tree based on the mitochondrial ND2 gene, including all species used for the positive selection analyses (see Supplementary Table 6 for species and GenBank accession numbers). We used the GTR $+\Gamma$ model with base frequencies and substitution rate matrix estimated from the data (as suggested by JMODELTEST ${ }^{42}$ ). We then ran CODEML implemented in PAML version $4.4 \mathrm{~b}$ to test for branch-specific adaptive evolution in $f h l 2 a$ and $f h l 2 b$ applying the branch-site model (free-ratios model with $\omega$ allowed to vary) $)^{59,60}$. The branch comparisons and results are shown in Supplementary Table 7.

Identification of CNEs. We then made use of the five available cichlid genomes ${ }^{61}$ to identify CNEs that could explain the difference in expression of $f h l 2 a$ and fhl $2 b$ between haplochromines and non-haplochromines (note that there are three haplochromine genomes available: A. burtoni, Pu. nyererei, $M$. zebra; and two genomes belonging to more ancestral cichlid lineages: $N$. brichardi and Or. niloticus). For this analysis, we also included the respective genomic regions of four other teleost species (O. latipes, Ta. rubripes, Te. nigroviridis and G. aculeatus). More specifically, we extracted the genomic scaffolds containing fhl $2 a$ and $f h l 2 b$ from the available cichlid genomes using BLAST v. 2.2.25 and the BIOCONDUCTOR R package BIOSTRINGS ${ }^{62}$ to extract 5-6 kb of sequence containing fhl2a and fhl2b from these scaffolds.

Comparative analyses of the $f h l 2 a$ and $f h l 2 b$ genomic regions were done with MVISTA (genome.lbl.gov/vista) ${ }^{63}$ using the LAGAN alignment tool ${ }^{64} ; A$. burtoni was used as a reference for the alignment. We applied the repeat masking option with $\mathrm{Ta}$. rubripes (Fugu) as reference. CNEs were defined as any non-coding section longer than $100 \mathrm{bp}$ that showed at least $70 \%$ sequence identity with A. burtoni.

Sequencing of the upstream region of $\mathbf{f h} / \mathbf{2 b}$. In order to confirm whether the AFC-SINE insertion was specific to egg-spot bearing haplochromines, we amplified the genomic region upstream of the fhl $2 b$ open reading frame in 19 additional cichlid species (10 haplochromines and 9 non-haplochromines). PCR amplification was performed as described above. For sequencing, we used four different primers, the two used in the amplification reaction and two internal primers, one haplochromine specific and another non-haplochromine specific. For detailed information about species and primers, see Supplementary Table 8.

Alignment of AFC-SINES from the $\boldsymbol{A}$. burtoni genome. SINE elements were identified using the SINE insertion sequence $5^{\prime}$ of the fhl $2 b$ gene of $A$. burtoni as query in a local BLASTn search ${ }^{49}$ with default settings against the $A$. burtoni reference genome. Blast hits were retrieved using custom scripts and extended to a region of 200-bp upstream and downstream of the identified sequence. Sequences were aligned using MAFFT v. 6 (ref. 41) with default settings and allowing for adjustment of sequence direction according to the reference sequence. The alignment was loaded into CODONCODE ALIGNER for manual correction and end trimming. Sequences shorter than $50 \mathrm{bp}$ were excluded from the alignment. The final alignment contained 407 sequences that were used to build the A. burtoni SINE consensus sequence using the consensus method implemented in CODONCODE ALIGNER with a percentage-based consensus and a cutoff of $25 \%$. The AFC-SINE element in the fhl $2 b$ promoter region was compared with the consensus sequence and available full-length AFC-SINE elements of cichlids in order to determine whether it was an insertion or deletion in haplochromines (Supplementary Table 8).

Characterization of fhl $\mathbf{2 b}$ upstream genomic region in cichlids. The $f h l 2 b$ genomic regions of the five cichlid genomes (A. burtoni, M. zebra, Pu. nyererei, N. brichardi, and O. niloticus) were loaded into CODONCODE ALIGNER and assembled (large gap alignments settings, identity cutoff 70\%). Assemblies were manually corrected. Transposable element sequences were identified using the Repeat Masking function of REPBASE UNIT (http://www.girinst.org/censor/ index.php) against all sequence sources and the bl2seq function of BLASTn ${ }^{49}$. Supplementary Fig. 6 shows a scheme of the transposable element composition of this genomic region in several cichlid species.

CNEs construct cloning and injection in zebrafish. We designed three genetic constructs containing the AFC-SINE and intron 1 of $f h l 2 b$ of three cichlid species (A. burtoni, P. philander and N. sexfasciatus) (Fig. 4) and one containing the $5^{\prime}$ untranslated region, exon 1 and intron 1 of $A$. burtoni fhl2a. The three fragments were amplified with PCR as described above (see Supplementary Table 3 for primer information). All fragments were cloned into a pCR8/GW/TOPO vector (Invitrogen) following the manufacturer's specifications. Sequence identity and direction of fragment insertion were confirmed via Sanger sequencing (as described above) using M13 primers. All plasmid extractions were performed with GenElute Plasmid Miniprep Kit (Sigma-Aldrich). We then recombined these fragments into the Zebrafish Enhancer Detection ZED vector ${ }^{65}$ following the protocol specified in ref. 66. Recombination into the ZED plasmid was performed taking into consideration the original orientation of the fhl $2 b$ genomic region. The resulting ZED plasmids were then purified with the DNA clean and concentrator -5 Kit (Zymo Research, USA). Injections were performed with $1 \mathrm{nl}$ into one-/two-cell stage zebrafish (D. rerio) embryos (A. burtoni construct was injected in wild-type strains $\mathrm{AB}$ and $\mathrm{ABxEK}, P$. philander and $N$. sexfasciatus constructs were injected

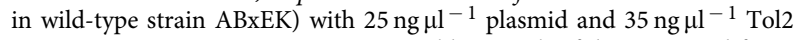
transposase mRNA. By outcrossing to wild-type zebrafish, we created five F2 stable transgenic lines for the A. burtoni construct, two F1 stable transgenic lines for the P. philander construct, and finally one F1 stable transgenic line for the N. sexfasciatus construct. Fish were raised and kept according to standard procedures ${ }^{67}$. Zebrafish were imaged using a Leica point scanning confocal microscope SP5-II-matrix and Zeiss LSM5 Pascal confocal microscope.

Fixation and dehydration of cichlid fins. In order to determine the pigment cell composition of egg-spots (and especially whether they contain iridophores in addition to xanthophores), we dissected A. burtoni anal fins. To better understand the morphological differences between non-haplochromine and haplochromine fins, we further dissected three $N$. crassus anal fins. To visualize iridophores, we removed the pteridine pigments of the overlying xanthophores by fixating the fin in $4 \%$ paraformaldehyde-PBS for $1 \mathrm{~h}$ at room temperature and washing it in a series of methanol:PBS dilutions $(25 \%, 50 \%, 75 \%$ and $100 \%)$. Pictures were taken after 6 days in $100 \%$ methanol at $-20^{\circ} \mathrm{C}$.

\section{References}

1. Pigliucci, M. What, if anything, is an evolutionary novelty? Philos. Sci. 75, 887-898 (2008).

2. Carroll, S. B. Evo-devo and an expanding evolutionary synthesis: a genetic theory of morphological evolution. Cell 134, 25-36 (2008).

3. Wagner, G. P. \& Lynch, V. J. Evolutionary novelties. Curr. Biol. 20, R48-R52 (2010).

4. Wagner, A. The molecular origins of evolutionary innovations. Trends Genet 27, 397-410 (2011)

5. Carroll, S. B., Grenier, J. K. \& Weatherbee, S. D. From DNA to Diversity: Molecular Genetics and the Evolution of Animal Design (Blackwell Science, 2001).

6. Schluter, D. Evidence for ecological speciation and its alternative. Science 323, 737-741 (2009).

7. Lynch, V. J., Leclerc, R. D., May, G. \& Wagner, G. P. Transposon-mediated rewiring of gene regulatory networks contributed to the evolution of pregnancy in mammals. Nat. Genet. 43, 1154-1159 (2011).

8. Beldade, P. \& Brakefield, P. M. The genetics and evo-devo of butterfly wing patterns. Nat. Rev. Genet. 3, 442-452 (2002).

9. Salzburger, W., Mack, T., Verheyen, E. \& Meyer, A. Out of Tanganyika: genesis, explosive speciation, key-innovations and phylogeography of the haplochromine cichlid fishes. BMC Evol. Biol. 5, 17 (2005).

10. Kocher, T. D. Adaptive evolution and explosive speciation: the cichlid fish model. Nat. Rev. Genet. 5, 288-298 (2004).

11. Salzburger, W. The interaction of sexually and naturally selected traits in the adaptive radiations of cichlid fishes. Mol. Ecol. 18, 169-185 (2009).

12. Fryer, G. \& Iles, T. The Cichlid Fishes of the Great Lakes of Africa: their Biology and Evolution (Oliver \& Boyd, 1972).

13. Wickler, W. 'Egg-dummies' as natural releasers in mouth-breeding cichlids Nature 194, 1092-1093 (1962).

14. Santos, M. E. \& Salzburger, W. How cichlids diversify. Science 338, 619-621 (2012).

15. Muschick, M., Indermaur, A. \& Salzburger, W. Convergent evolution within an adaptive radiation of cichlid fishes. Curr. Biol. 22, 2362-2368 (2012).

16. Salzburger, W., Braasch, I. \& Meyer, A. Adaptive sequence evolution in a color gene involved in the formation of the characteristic egg-dummies of male haplochromine cichlid fishes. BMC Biol. 5, 51 (2007).

17. Braasch, I. \& Postlethwait, J. in Polyploidy and Genome Evolution (eds Soltis, D E. \& Soltis, P. S.) 341-383 (Springer, 2012). 
18. Müller, J. M. et al. FHL2, a novel tissue-specific coactivator of the androgen receptor. EMBO J. 19, 359-369 (2000).

19. Brun, J. et al. The LIM-only protein FHL2 controls osteoblast mesenchymal cell differentiation through non-canonical Wnt signalling. Bone 50, S76 (2012).

20. Johannessen, M., Møller, S., Hansen, T., Moens, U. \& Van Ghelue, M. The multifunctional roles of the four-and-a-half-LIM only protein FHL2. Cell. Mol. Life Sci. 63, 268-284 (2006).

21. Kadrmas, J. L. \& Beckerle, M. C. The LIM domain: from the cytoskeleton to the nucleus. Nat. Rev. Mol. Cell Biol. 5, 920-931 (2004).

22. Heule, C. \& Salzburger, W. The ontogenetic development of egg-spots in the haplochromine cichlid fish Astatotilapia burtoni. J. Fish Biol. 78, 1588-1593 (2011).

23. Baldo, L., Santos, M. E. \& Salzburger, W. Comparative transcriptomics of Eastern African cichlid fishes shows signs of positive selection and a large contribution of untranslated regions to genetic diversity. Genome Biol. Evol. 3, 443-455 (2011)

24. Lynch, M. \& Conery, J. S. The evolutionary fate and consequences of duplicate genes. Science 290, 1151-1155 (2000).

25. Takahashi, K., Terai, Y., Nishida, M. \& Okada, N. A novel family of short interspersed repetitive elements (SINEs) from cichlids: the patterns of insertion of SINEs at orthologous loci support the proposed monophyly of four major groups of cichlid fishes in Lake Tanganyika. Mol. Biol. Evol. 15, 391-407 (1998).

26. Britten, R. J. \& Davidson, E. H. Gene regulation for higher cells: a theory. Science 165, 349-357 (1969)

27. Britten, R. J. \& Davidson, E. Repetitive and non-repetitive DNA sequences and a speculation on the origins of evolutionary novelty. Q Rev. Biol. 46, 111-138 (1971).

28. Feschotte, C. Transposable elements and the evolution of regulatory networks. Nat. Rev. Genet. 9, 397-405 (2008).

29. Frohnhöfer, H. G., Krauss, J., Maischein, H.-M. \& Nüsslein-Volhard, C. Iridophores and their interactions with other chromatophores are required for stripe formation in zebrafish. Development 140, 2997-3007 (2013).

30. Patterson, L. B. \& Parichy, D. M. Interactions with iridophores and the tissue environment required for patterning melanophores and xanthophores during zebrafish adult pigment stripe formation. PLoS Genet. 9, e1003561 (2013).

31. Manousaki, T. et al. Parsing parallel evolution: ecological divergence and differential gene expression in the adaptive radiations of thick-lipped Midas cichlid fishes from Nicaragua. Mol. Ecol. 22, 650-669 (2013).

32. Shimeld, S. M. \& Holland, P. W. Vertebrate innovations. Proc. Natl Acad. Sci. USA 97, 4449-4452 (2000).

33. Egger, B., Klaefiger, Y., Theis, A. \& Salzburger, W. A sensory bias has triggered the evolution of egg-spots in cichlid fishes. PLOS ONE 6, e25601 (2011)

34. Theis, A., Salzburger, W. \& Egger, B. The function of anal fin egg-spots in the cichlid fish Astatotilapia burtoni. PLoS ONE 7, e29878 (2012)

35. Miller, S., Dykes, D. \& Polesky, H. A simple salting out procedure for extracting DNA from human nucleated cells. Nucleic Acids Res. 16, 1215 (1988).

36. Meyer, B. S. \& Salzburger, W. A novel primer set for multilocus phylogenetic inference in East African cichlid fishes. Mol. Ecol. Resour. 12, 1097-1104 (2012).

37. Margulies, M. et al. Genome sequencing in microfabricated high-density picolitre reactors. Nature 437, 376-380 (2005)

38. Binladen, J. et al. The use of coded PCR primers enables high-throughput sequencing of multiple homolog amplification products by 454 parallel sequencing. PLoS ONE 2, e197 (2007).

39. Schmieder, R. \& Edwards, R. Quality control and preprocessing of metagenomic datasets. Bioinformatics 27, 863-864 (2011).

40. Kearse, M. et al. Geneious Basic: an integrated and extendable desktop software platform for the organization and analysis of sequence data. Bioinformatics 28, 1647-1649 (2012).

41. Katoh, K., Kuma, K., Toh, H. \& Miyata, T. MAFFT version 5: improvement in accuracy of multiple sequence alignment. Nucleic Acids Res. 33, 511-518 (2005)

42. Darriba, D., Taboada, G. L., Doallo, R. \& Posada, D. jModelTest 2: more models, new heuristics and parallel computing. Nat. Methods $\mathbf{9}, 772$ (2012).

43. Schwarz, G. Estimating the dimension of a model. Ann. Stat. 6, 461-464 (1978).

44. Ronquist, F. et al. MrBayes 3.2: efficient Bayesian phylogenetic inference and model choice across a large model space. Syst. Biol. 61, 539-542 (2012).

45. Zwickl, D. J. Genetic Algorithm Approaches for the Phylogenetic Analysis of Large Biological Sequence Datasets Under the Maximum Likelihood Criterion (University of Texas, 2006).

46. Sukumaran, J. \& Holder, M. T. DendroPy: a Python library for phylogenetic computing. Bioinformatics 26, 1569-1571 (2010).
47. Li, H. et al. The Sequence Alignment/Map format and SAMtools. Bioinformatics 25, 2078-2079 (2009).

48. Robinson, M. D., McCarthy, D. J. \& Smyth, G. K. edgeR: a Bioconductor package for differential expression analysis of digital gene expression data. Bioinformatics 26, 139-140 (2010).

49. Altschul, F. S., Gish, W., Miller, W., Myers, W. E. \& Lipman, J. D. Basic Local Alignment Search Tool. J. Mol. Biol. 215, 403-410 (1990).

50. Pfaffl, M. W. A new mathematical model for relative quantification in real-time RT-PCR. Nucleic Acids Res. 29, e45 (2001).

51. Curran, K. et al. Interplay between Foxd3 and Mitf regulates cell fate plasticity in the zebrafih neural crest. Dev. Biol. 344, 107-118 (2010).

52. Tang, R., Dodd, A., Lai, D., McNabb, W. C. \& Love, D. R. Validation of zebrafish (Danio rerio) reference genes for quantitative real-time RT-PCR normalization. Acta. Biochim. Biophys. Sin. 39, 384-390 (2007).

53. Zhang, Z. \& Hu, J. Development and validation of endogenous reference genes for expression profiling of medaka (Oryzias latipes) exposed to endocrine disrupting chemicals by quantitative real-time RT-PCR. Toxicol. Sci. 95, 356-368 (2007).

54. Catchen, J. M., Conery, J. S. \& Postlethwait, J. H. Automated identification of conserved synteny after whole-genome duplication. Genome Res. 19, 1497-1505 (2009).

55. Rozen, S. \& Skaletsky, H. Primer3 on the WWW for general users and for biologist programmers. Methods Mol. Biol. 132, 365-386 (2000).

56. Flicek, P. et al. Ensembl 2012. Nucleic Acids Res. 40, D84-D90 (2012).

57. Larkin, M. et al. Clustal W and Clustal X version 2.0. Bioinformatics 23, 2947-2948 (2007).

58. Swofford, D. L. PAUP*. Phylogenetic Analysis Using Parsimony ( ${ }^{*}$ and Other Methods) (Sinauer, 2003).

59. Yang, Z. PAML 4: phylogenetic analysis by maximum likelihood. Mol. Biol. Evol. 24, 1586-1591 (2007).

60. Nielsen, R. \& Yang, Z. Likelihood models for detecting positively selected amino acid sites and applications to the HIV-1 envelope gene. Genetics 148, 929-936 (1998)

61. Brawand, D. et al. The genomic substrate for adaptive radiation in Africa cichlid fish. Nature 513, 375-381 (2014).

62. Pages, H., Aboyoun, P., Gentleman, R. \& DebRoy, S. Biostrings: String objects representing biological sequences, and matching algorithms. R-package, http:// www.bioconductor.org/packages/release/bioc/html/Biostrings.html.

63. Frazer, K. A., Pachter, L., Poliakov, A., Rubin, E. M. \& Dubchak, I. VISTA: computational tools for comparative genomics. Nucleic Acids Res. 32, W273-W279 (2004)

64. Brudno, M. et al. LAGAN and Multi-LAGAN: efficient tools for large-scale multiple alignment of genomic DNA. Genome Res. 13, 721-731 (2003).

65. Bessa, J. et al. Zebrafish enhancer detection (ZED) vector: a new tool to facilitate transgenesis and the functional analysis of cis-regulatory regions in zebrafish. Dev. Dyn. 238, 2409-2417 (2009).

66. Bessa, J. \& Gómez-Skarmeta, J. L. Making reporter gene constructs to analyze cis -regulatory elements. Mol. Methods Evol. Genet. 772, 397-408 (2011).

67. Westerfield, M. The Zebrafish Book. A guide for the Laboratory Use of Zebrafish (Danio rerio) (University of Oregon Press, 2000).

\section{Acknowledgements}

We thank A. Theis, T. Bosia, B. Egger, G. Banyankimbona, E. Schraml, M. Muschick and H. Gante for their assistance with the photographs; P. Beldade, J. Ganz, H. Hoekstra, J.H Postlethwait, G. Wagner and the members of the Salzburger Laboratory for their discussions; J. Bessa for sending us the ZED vector; V. Laudet for providing medaka and zebrafish; J. Ganz, University of Oregon and the Imaging Core Facility of the Biozentrum, University of Basel for their support with confocal microscopy; the University of Oregon zebrafish facility, A. Indermaur and A. Rüegg (Basel) for fishkeeping; and the BROAD Institute and the Cichlid Genome Consortium for the cichlid genomes. This study was supported by grants from the Fundação para a Ciência e Tecnologia (FCT, Portugal) to M.E.S.; the Volkswagen Stiftung to I.B. and A.B.; the 'Fellowship for Excellence' from the International PhD Program in Molecular Life Sciences and the Werner-Siemens-Foundation (Aug) to L.S.; the Swiss National Science Foundation (SNF) to M.A.; and the European Research Council (Starting Grant 'INTERGENADAPT'), the University of Basel, the National Geographic Society and the SNF to W.S. The work of I.B. was further supported by National Institutes of Health grant R01 RR020833 (R01 OD011116) to John H. Postlethwait.

\section{Author contributions}

M.E.S., I.B. and W.S. designed the study; M.E.S and W.S. collected the samples; M.E.S. performed the RNAseq, gene expression, comparative genomics and zebrafish functional analysis; N.B. performed the sequencing of $f h l 2$ paralogs coding region and analysed its rates of evolution; B.S.M. collected the 454 sequence data; and B.S.M. and W.S. performed the phylogenetic analysis; A.B. performed the SINE consensus alignments and analysed the transposable element composition of $f h l 2 b$ genomic region; I.B. performed the zebrafish functional assays of the $A$. burtoni construct and $f h l 2$ paralogs synteny 
analysis; L.S., H.-G.B. and M.A. assisted with the zebrafish functional assays of the A. burtoni, P. philander and N. sexfasciatus construct; and M.E.S. and W.S. wrote the paper and all authors contributed to revisions.

\section{Additional information}

Accession codes: All nucleotide sequences reported in this study have been deposited in GenBank/EMBL/DDBJ under the accession codes KM263618 to KM264016. All the short reads have been deposited in GenBank/EMBL/DDBJ Sequence Read Archive (SRA) under the BioProject ID PRJNA25755.

Supplementary Information accompanies this paper at http://www.nature.com/ naturecommunications

Competing financial interests: The authors declare no competing financial interests.
Reprints and permission information is available online at http://npg.nature.com/ reprintsandpermissions/

How to cite this article: Santos, M. E. et al. The evolution of cichlid fish egg-spots is linked with a cis-regulatory change. Nat. Commun. 5:5149 doi: 10.1038/ncomms6149 (2014)

(c) (1) This work is licensed under a Creative Commons Attributioncc. ${ }_{\mathrm{BY}}$ NC SA NonCommercial-ShareAlike 4.0 International License. The images or other third party material in this article are included in the article's Creative Commons license, unless indicated otherwise in the credit line; if the material is not included under the Creative Commons license, users will need to obtain permission from the license holder to reproduce the material. To view a copy of this license, visit http:// creativecommons.org/licenses/by-nc-sa/4.0/ 